\title{
GAN Based Noise Generation to Aid Activity Recognition when Augmenting Measured WiFi Radar Data with Simulations
}

\author{
Shelly Vishwakarma*, Chong Tang*,Wenda $\mathrm{Li}^{*}$, Karl Woodbridge ${ }^{\dagger}$, Raviraj Adve ${ }^{\ddagger}$, Kevin Chetty* \\ ${ }^{*}$ Department of Security and Crime Science, University College London, UK \\ ${ }^{\dagger}$ Department of Electronic and Electrical Engineering, University College London, UK \\ $\ddagger$ Department of Electrical and Computer Engineering, University of Toronto, Canada \\ \{s.vishwakarma, chong.tang.18, wenda.li, k.woodbridge, k.chetty\}@ucl.ac.uk, rsadve@comm.utoronto.ca
}

\begin{abstract}
This work considers the use of a passive WiFi radar (PWR) to monitor human activities. Real-time uncooperative monitoring of people has numerous applications ranging from smart cities and transport to IoT and security. In e-healthcare, PWR technology could be used for ambient assisted living and early detection of chronic health conditions. Large training datasets could drive forward machine-learning-focused research in the above applications. However, generating and labeling large volumes of high-quality, diverse radar datasets is an onerous task. Therefore, we present an open-source motion capture data-driven simulation tool, SimHumalator, that can generate large volumes of human micro-Doppler radar data at multiple IEEE WiFi standards(IEEE 802.11g, $\mathrm{n}$, and ad). We qualitatively compare the micro-Doppler signatures generated through SimHumalator with the measured signatures. To create a more realistic training dataset, we artificially add noise to our clean simulated spectrograms. A noise distribution is directly learned from real radar measurements using a Generative Adversarial Network (GAN). We observe improvements in the classification performances between 3 to $8 \%$. Our results suggest that simulation data can be used to make adequate training data when the available measurement training support is low.

Index Terms-Passive WiFi Sensing, micro-Doppler, activity recognition, generative adversarial networks
\end{abstract}

\section{INTRODUCTION}

A significant growth in an aging population and the number of people with long-term disability and chronic health conditions has necessitated the development of technologies enabling healthy living such as activity monitoring systems, wearable sensors, etc. [1]. RF sensing has received increased attention among various technologies, as it is minimally invasive, privacy-preserving, and can continuously monitor human activities remotely. It is low-cost and typically easy to deploy using commercial off-the-shelf (COTS) components.

When illuminated by a radio-frequency (RF) source, human hands and legs' movement gives rise to frequency variations along the main body Doppler (popularly known as micro-Dopplers). Micro-Dopplers possess unique and discriminative features when observed in joint time-frequency space [2]. Extensive researches have exploited these signatures for indoor healthcare applications such as assisted living [3], and bio-medical applications (for non-intrusively monitoring patients) [4].

Micro-Dopplers have been captured using both active and passive RF sensors. Active sensing uses the system's own transmissions as a source of illumination, whereas passive sensing leverages existing opportunistic signals such as WiFi (or other wireless) communications to capture RF returns passively [5]. Due to the number of advantages, such as its receive only nature, low energy consumption, and the possible presence of multiple illuminators, passive sensing systems have become increasingly popular [5], [6]. All these works' essential functionality has been human activity classification using deep learning assisted solutions.

Training any deep learning model, however, requires high volumes of good quality labeled data. The amount of data captured in radars are often limited due to the following reasons: first, collecting real-world micro-Doppler data can be a very laborious and costly task; Second, the data is affected by various environmental factors, sensor parameters, and target characteristics. There have been significant efforts to overcome the constraints associated with the limited measurement databases. The work in [7], [8], used data augmentation technique to generate more extensive databases through operations such as flipping, time-shifting, or using generative adversarial networks (GANs).

Our previous work presented an open-source simulation tool for the passive WiFi radar (PWR) scenario, called SimHumalator [9] where we leveraged human micro-Doppler data generated using SimHumalator to augment our measured data [10]. The results highlight that augmentation can improve the classification performance when only limited experimental data is available for training. However, the simulated spectrograms considered in our previous study did not consider environmental factors such as noise, occlusions, multipath, and propagation loss. Most realistic scenarios certainly have factors that significantly affect the resulting micro-Doppler signatures.

In this study, we artificially add two types of noise to our clean simulated spectrograms. The first is additive white Gaussian noise (AWGN) which is always present in the measurement data. AWGN is pixel-independent, spatially uncorrelated, but does not incorporate practical phenomena such as clutter and multipath. Therefore, to mimic complex real-world scenarios, we propose a second type of noise modeling method based on a GAN. We use a GAN to 


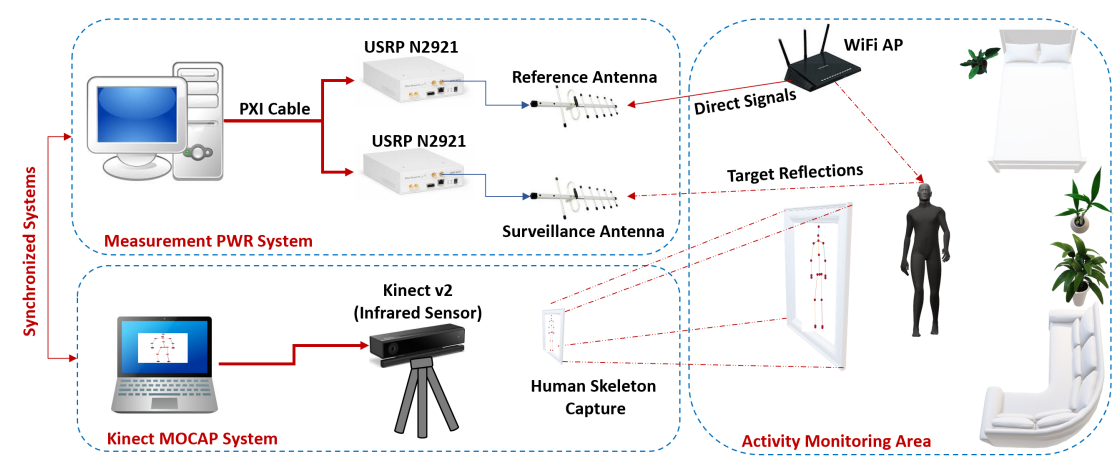

Fig. 1: The experimental setup comprising two synchronized systems- a motion capture Kinect v2 sensor and a PWR system for monitoring human activities in indoor scenarios.

learn noise distribution models directly from the measured spectrograms. We then add this realistic noise signal to our clean simulated spectrograms.

We apply our approach to perform a detailed augmentation study using three simulation datasets- one with no noise, the second with added AWGN noise, and finally with GAN-based noise. To sum up, our contributions in this paper are the following:

1) We use synthetic signatures generated by SimHumalator for data augmentation to solve the practical problem associated with limited or unbalanced microDoppler training datasets.

2) We use a GAN to learn noise directly from measurement data, considering factors such as clutter, noise, and multipath. We then add this noise to simulated data to enable a more accurate representation of the sensing scenario.

3) Finally, we study two data augmentation methodsReplacement and Augmentation and validate their performance in different scenarios using a convolutional neural network $(\mathrm{CNN})$.

We observe an increase in average classification accuracy between 3\% to $8 \%$ for GAN-generated noise, which suggests that simulation data can be used to improve recognition without the burden of collecting significant quantities of experimental training data.

Our paper is organized as follows. Section II describes the experimental setup, data collection, and the proposed noise modeling framework based on the GANs. Section III shows interesting classification scenarios where we augment measurement data with simulation data under - no-noise conditions, AWGN noise, and GAN generated noise. We finally conclude our paper in Section IV.

\section{Experimental Setup And Data Collection}

Fig. 1 presents our experimental setup comprising two synchronized systems: an infrared motion capture Kinect v2 sensor and a non-contact physical activity monitoring PWR system.

\section{A. Experimental System Framework}

The PWR system is set up using two Yagi antennas, each with a gain of $14 \mathrm{dBm}$, two National Instruments (NI) USRP-

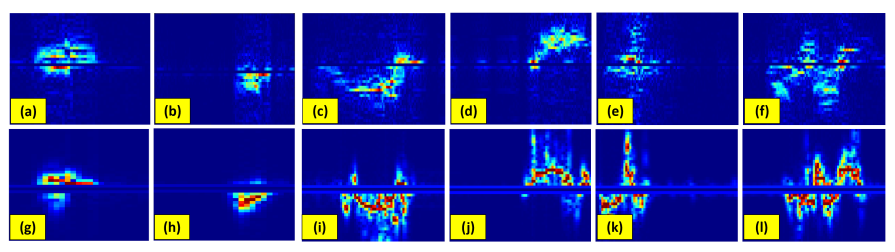

Fig. 2: (a)-(f) presents measured spectrograms and (g)-(l) simulated spectrograms corresponding to a human six different activities: siting-down on a chair, standing up from a chair, walk-to-sit, sit-towalk, walk to fall-down, and stand-from-floor to walk, respectively.

2921 [11] and a Netgear R6300 transmitter acting as the WiFi AP. We configure the WiFi AP to transmit a $802.11 \mathrm{~g}$ standardcompliant waveform at a center frequency of $2.472 \mathrm{GHz}$. The PWR system uses one antenna, a reference antenna, to capture direct WiFi transmissions from the AP. Simultaneously, the second is a surveillance antenna to capture signals reflected off targets in the propagation environment. The reference WiFi signal and the surveillance signal are then cross-correlated to generate the radar micro-Doppler signatures in real-time.

Kinect v2 sensor, on the other hand, being a depth camera captures three-dimensional (3D) information of the joints on the human body [12], [13]. We use our open-source simulation tool SimHumalator to generate radar micro-Doppler signatures from the Kinect animation data [9]. SimHumalator simulates an IEEE 802.11g standard-compliant WiFi signal using MATLAB's WLAN toolbox and combines it with animation data to simulate the reflected signals. We refer the readers to [9] for a detailed description of the SimHumalator working methodology.

Since we are capturing IEEE 802.11g WiFi transmissions, the signal bandwidth is limited to $20 \mathrm{MHz}$, which is insufficient to locate targets in most indoor scenarios. Therefore, we focus on extracting only the time-varying micro-Doppler information in joint time-frequency space.

\section{B. Data Collection}

We monitor three participants (two males and one female) performing six different activities: (i) siting-down on a chair, (ii) standing up from a chair, (iii) walk-to-sit, (iv) sit-towalk, (v) walk to fall-down, and (vi) stand-from-floor to walk. This study restricts our measurements to direct line-of-sight 


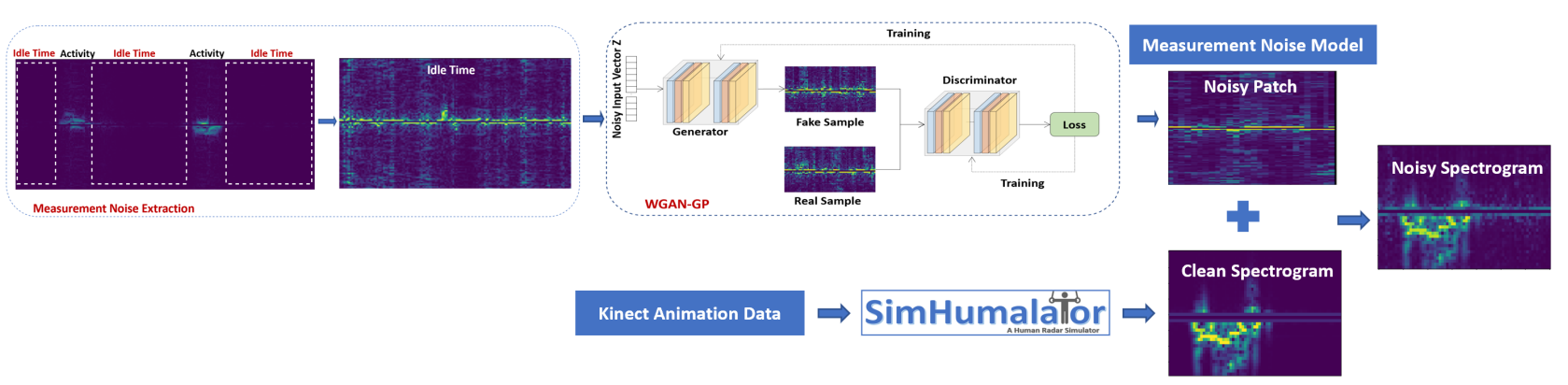

Fig. 3: Noise modeling framework based on GANs

conditions with targets moving between $0.8 \mathrm{~m}$ to $3.8 \mathrm{~m}$ in front of the system. Each activity is recorded for $5 \mathrm{sec}$. We repeated the measurements 20 times for each participant, resulting in 360 total measurements. We simultaneously recorded 360 animation files.

Fig. 2 shows the qualitative comparison between the micro-Doppler spectrograms generated using the PWR measurement system and the SimHumalator for each of the six activities. Fig. 2 (a)-(f) corresponds to the measured spectrogram while Fig. 2 (g)-(i) are the corresponding simulated spectrograms. We can observe a close similarity between the simulated spectrograms and the measured spectrograms. The spectrograms' signals' strength differs, likely because our simulations do not synthesize environmental factors like noise, propagation loss, occlusions, and multipath.

\section{Spectrograms Evaluation Under Noisy Conditions}

We add noise to our clean simulated spectrogram to mimic a more realistic scenario and use noisy signatures for data augmentation to improve the classification performance. Most studies assume the noise model to be AWGN [14], [15]. This preference, however, has some limitations. AWGN does account for factors such as multipath and clutter. As a result, inspired by our denoising study [16], we adopt a GANbased measurement noise model (MNM), where we learn the model directly from real measurements to generate a more realistic noise. Fig. 3 presents the proposed GAN-based noise simulation framework.

- Step1: Measurement Noise Extraction: The primary step is the noise extraction from the measured spectrograms. We split our activity monitoring periods into-Idle time (when there is no activity) and the second period when a human is undergoing a particular activity. We extract noise patches from the idle time zones as it is hard to separate noise from the regions that contain target motion characteristics.

- Step2: Training GAN: We use WGAN-GP [17] as it can effectively overcome the gradient disappearance and training instability issues that hinder traditional GANs. GAN includes a generator model, and a discriminator model. The generator aims to fool the discriminator by mimicking the distribution of the measurement noise. By contrast, the discriminator first learns the distribution from the measured samples, and then examines how close the generated distribution and the distribution of the measurement noise are, providing a reality score. Finally, the reality score is fed back to the generator and discriminator to adjust the network parameters until the network converges. Learning a measurement noise model fundamentally involves minimizing the objective function

$L=E_{\tilde{x} \sim p_{g}}[D(x)]-E_{x \sim p_{r}}[D(x)]+\lambda E_{\hat{x} \sim p_{\hat{x}}}\left[\left(\left\|\nabla_{\hat{x}} D(\hat{x})\right\|_{2}-1\right)^{2}\right]$

where $p_{r}$ represents the data distribution over the measurement noise, $p_{g}$ is the noise distribution learned by the generator and $p_{\hat{x}}$ is uniformly sampled along straight lines between pairs of points sampled from $p_{r}$ and $p_{g}$. Note that the inner-structure of the generator and discriminator uses convolutional neural networks (CNNs). We refer the readers to [16] for detailed description of network architecture.

- Step3: SNR Fixation: In practical scenarios, we normally evaluate the noise level by the signal-to-noise ratio (SNR), which can be calculated as:

$$
S N R=10 * \log 10\left(\frac{\sigma_{\text {signals }}^{2}}{\sigma_{\text {noise }}^{2}}\right)
$$

where $\sigma^{2}$ represent the variance of data. For a given noise patch, we can control $\sigma_{\text {noise }}^{2}$ by multiplying the noise patch by a factor, $\alpha$. To obtain $\alpha$, we first deduce the desired variance of the noise patch, $\sigma_{\text {noise }}^{2}$, from the given SNR value and 2. Second, we calculate the original variance of the noise patch as $\sigma_{\text {noise }}^{2}$. We finally compute $\alpha=\operatorname{sqr} t\left(\frac{\sigma_{\text {noise }}^{2}}{\sigma_{\text {noise }}^{2}}\right)$ and add the noisy patch to our clean spectrograms.

Fig. 4 presents the synthetic spectrograms with varying SNR levels for a human undergoing a motion of sitting down on a chair. Fig. 4(a)-(c) corresponds to spectrograms with added AWGN noise and Fig. 4(d)-(f) shows the spectrograms added with MNM noise for three SNRs-0dB, 9dB, and 21dB respectively. We can observe that the noise distribution in Fig. 4(d)-(f) is close to the noise distribution of the measured spectrogram thus is more realistic compared to the spectrograms added with AWGN noise. 

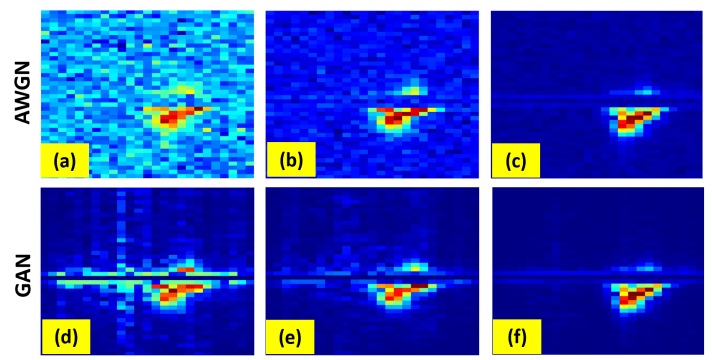

Fig. 4: Noisy simulated spectrograms with: (a)-(c) AWGN noise, (d)(f) GAN generated MNM noise for three SNRs- $0 \mathrm{~dB}, 9 \mathrm{~dB}$ and $21 \mathrm{~dB}$ respectively.

III. EXPERImental Results AND ANALYsis

We investigate the data augmentation performance in four different scenarios.

- Case 1:Both training and test dataset comprise measurement data only (TMTM).

- Case 2: Training dataset created by combining the clean simulation data and measurement data.

- Case 3: Training dataset created by combining the simulation data with added AWGN noise and measurement data.

- Case 4: Training dataset created by combining the simulation data with added MNM noise and measurement data.

We split the total measurements dataset $(M=360)$ into two $50 \%$ for training and $50 \%$ for the test, resulting in a measurement data split ratio of $m=0.5$. We used an eight-layered convolutional neural network (CNN) to test the classification performance. Each spectrogram's size is fixed to 100 by 100; the batch size is set to 32; the adaptive moment estimation optimizer's learning rate is set to 0.01 , and the epoch is set to 150 . We kept identical training parameters for all the cases under consideration and repeated the experiments ten times. We finally report the average classification accuracy achieved in each scenario. Note the average classification accuracy when the training and test datasets comprise measurement data only is $92.7 \%$.

\section{A. Replacement Study}

Fig. 5 presents a replacement study where we replace a percentage of measurement data with simulation data in the training dataset. $s$ is the percentage of replacement. Fig. 6 presents the classification accuracy as a function of the varying percentage of replacement $s$ for two SNRs$0 \mathrm{~dB}, 21 \mathrm{~dB}$ and for two noise scenarios- AWGN and MNM noise. We can observe that MNM noise performance is higher than simulation data with AWGN noise for both SNRs $(\approx 8.5 \%$ when $s=90 \%$ ). The classification accuracy although decreases with an increase of $s$, which is due to the inherent differences between measurement data and simulated data. For a practical system up to $50 \%$, corrupted measurement data can be replaced with simulation data before classification performance becomes unreliable. Table I report the classification accuracy for: simulation data with no noise, with AWGN noise and MNM noise as a function of varying SNR. Some of the observations from the study are:

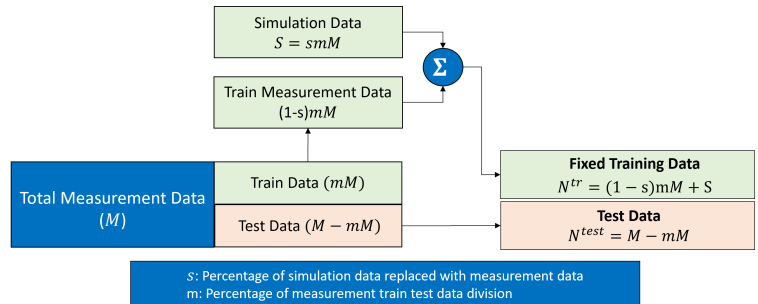

Fig. 5: Replacement study: The ratio of measurement data replaced with simulation data is varied to study the impact of data replacement on the classification performance. $s=0$, is a special case where both training and test data comprise of measured spectrograms only (TMTM).

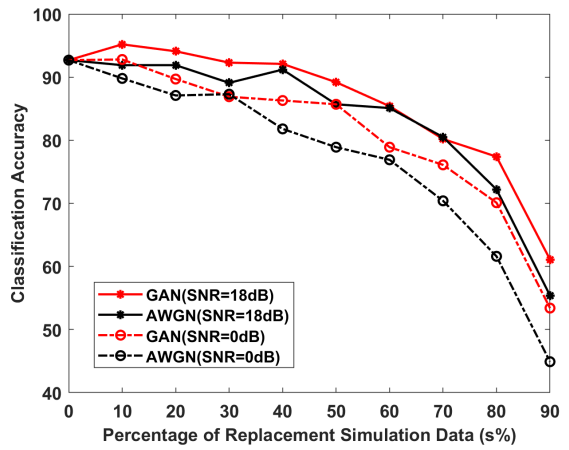

Fig. 6: Classification accuracies as a function of percentage of replacement data for two noise models: AWGN and MNM noise for two SNRs- 0dB and $21 \mathrm{~dB}$.

- Under low SNR scenarios, the performance of simulation data with MNM noise is higher compared to data with AWGN noise for almost all $s$. This is because the AWGN signal corrupts the micro-Doppler signatures to the extent where it is hard to extract the discriminating feature, resulting in reduced classification performance.

- For a specific $s$, the performance improves until a certain SNR is reached. This indicates that the SNR of the measured data is likely to be within this range.

- For almost all cases, the data's performance with MNM noise is higher than simulation data with either no noise or AWGN noise. We have highlighted the best performing cases in red for all SNRs and each $s$.

We believe that the replacement with MNM noise data can significantly improve classification accuracy compared to no noise and AWGN noise cases under low measurement training support.

\section{B. Augmentation Study}

In this study, $s$ percentage of the simulation data $S$ is added to the entire measurement training data $m M$ resulting in increased training support of size $(m M+s S)$. The study is summarized in Fig. 7. Like the replacement study, we present classification accuracies in Fig. 8 for two SNRs- 0dB and $21 \mathrm{~dB}$. The classification performance almost remains constant for all the $s$ and both noisy datasets. However, MNM noisy data's performance is better than data with AWGN noise. It is also important to note that augmentation is $3 \%$ better than when $s=0$. TABLE II presents the augmentation 
TABLE I: Classification accuracy results for replacement study. $\mathrm{m}$ is fixed to $50 \%$. $\mathrm{s}$ is varied from $0 \%$ to $90 \%$ and SNR is varied from $0 \mathrm{~dB}$ to $21 \mathrm{~dB}$ with an interval of $3 \mathrm{~dB}$. The best performing cases for all SNRs and each $s$ are highlighted in red color.

\begin{tabular}{|c|c|c|c|c|c|c|c|c|c|}
\hline \multicolumn{2}{|c|}{ Replacement Study (Measurement data split 50\%-50\%) } & \multicolumn{8}{|c|}{ SNR(dB) } \\
\hline Percentage of Measurement Data Replaced & Noise Model & 0 & 3 & 6 & 9 & 12 & 15 & 18 & 21 \\
\hline \multirow{3}{*}{$90 \% \mathrm{M}+10 \% \mathrm{~S}$} & No Noise & 92.8 & 92.8 & 92.8 & 92.8 & 92.8 & 92.8 & 92.8 & 92.8 \\
\hline & AWGN & 89.8 & 90.4 & 92.4 & 92.4 & 92.7 & 93.9 & 91.9 & 94 \\
\hline & GAN Noise & 92.8 & 92.1 & 92.5 & 92.7 & 92.6 & 95.1 & 95.2 & 94.5 \\
\hline \multirow{3}{*}{$80 \% \mathrm{M}+20 \% \mathrm{~S}$} & No Noise & 91.2 & 91.2 & 91.2 & 91.2 & 91.2 & 91.2 & 91.2 & 91.2 \\
\hline & AWGN & 87.1 & 88.1 & 90.7 & 91.1 & 91.2 & 91.9 & 91.9 & 93.2 \\
\hline & GAN Noise & 89.7 & 91.8 & 92.2 & 93.6 & 92.8 & 92.6 & 94.1 & 93.4 \\
\hline \multirow{3}{*}{$70 \% \mathrm{M}+30 \% \mathrm{~S}$} & No Noise & 87.3 & 87.3 & 87.3 & 87.3 & 87.3 & 87.3 & 87.3 & 87.3 \\
\hline & AWGN & 87.3 & 85.9 & 88.2 & 87.2 & 89.3 & 92.2 & 89.1 & 90.8 \\
\hline & GAN Noise & 86.9 & $\begin{array}{l}89.3 \\
\end{array}$ & 91.8 & 92.7 & 90.6 & 92.2 & 92.3 & 91.9 \\
\hline \multirow{3}{*}{$60 \% \mathrm{M}+40 \% \mathrm{~S}$} & No Noise & 89.3 & 89.3 & 89.3 & 89.3 & 89.3 & 89.3 & 89.3 & 89.3 \\
\hline & AWGN & 81.8 & 82.8 & 87.9 & 86.6 & 86.2 & 90.6 & 91.2 & 88.9 \\
\hline & GAN Noise & 86.3 & 88.1 & 89.7 & 91.4 & 90.1 & 89.1 & 92.1 & 90.5 \\
\hline \multirow{3}{*}{$50 \% \mathrm{M}+50 \% \mathrm{~S}$} & No Noise & 83.3 & 83.3 & 83.3 & 83.3 & 83.3 & 83.3 & 83.3 & 83.3 \\
\hline & AWGN & 78.9 & 79.3 & 85.3 & 85.1 & 86.6 & 85.4 & 85.7 & 85.7 \\
\hline & GAN Noise & 85.7 & 86.6 & 88.3 & 87.7 & 89.1 & 88.1 & 89.2 & 87.3 \\
\hline \multirow{3}{*}{$40 \% \mathrm{M}+60 \% \mathrm{~S}$} & No Noise & 78.1 & 78.1 & 78.1 & 78.1 & 78.1 & 78.1 & 78.1 & 78.1 \\
\hline & AWGN & 76.9 & 82 & 78.9 & 83.4 & 83.7 & 85.1 & 85.1 & 83.3 \\
\hline & GAN Noise & 78.9 & 81.8 & 84.3 & 85.8 & 85.7 & 86.6 & 85.4 & 88.7 \\
\hline \multirow{3}{*}{$30 \% \mathrm{M}+70 \% \mathrm{~S}$} & No Noise & 71.4 & 71.4 & 71.4 & 71.4 & 71.4 & 71.4 & 71.4 & 71.4 \\
\hline & AWGN & 70.4 & 75.4 & 78 & 77.8 & 79.1 & 78.3 & 80.5 & 82.1 \\
\hline & GAN Noise & 76.1 & 78.9 & 80.3 & 81.9 & 79.5 & 83.8 & 80.2 & 82.2 \\
\hline \multirow{3}{*}{$20 \% M+80 \% S$} & No Noise & 67.9 & 67.9 & 67.9 & 67.9 & 67.9 & 67.9 & 67.9 & 67.9 \\
\hline & AWGN & 61.6 & 65.2 & 71.2 & 69.9 & 71.6 & 73.6 & 72.2 & 70.3 \\
\hline & GAN Noise & 70.1 & 68.8 & 72.3 & 72.4 & 74.2 & 72.6 & 77.4 & 75.7 \\
\hline \multirow{3}{*}{$10 \% \mathrm{M}+90 \% \mathrm{~S}$} & No Noise & 51.7 & 51.7 & 51.7 & 51.7 & 51.7 & 51.7 & 51.7 & 51.7 \\
\hline & AWGN & 44.9 & 49.3 & 54.5 & 54.6 & 54.9 & 55.1 & 55.4 & 51.3 \\
\hline & GAN Noise & 53.4 & 53.3 & 54.9 & 54.3 & 58.6 & 60.8 & 61.1 & 60.6 \\
\hline
\end{tabular}

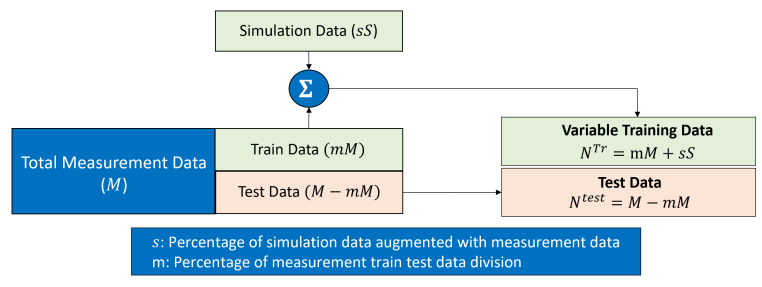

Fig. 7: Augmentation study: The training dataset changes with the percentage of simulated data (s) augmented with the measurement dataset.

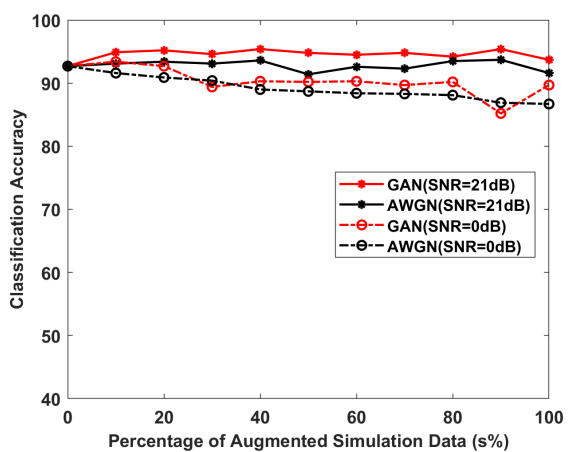

Fig. 8: Classification accuracies as a function of the percentage of augmented data for two noise models: AWGN and MNM noise for two SNRs- 0dB and $21 \mathrm{~dB}$.

results as a function of SNR and percentage of data augmentation for three cases- no noise, data with AWGN noise, and data with MNM noise. The classification accuracies remain almost constant with an increase of $s$ but better than the $s=0$ case. The possible reason is increased training support.
At low SNRs, the performance for both data with MNM noise and AWGN noise drops compared to when $s=0$. The drop is higher for AWGN due to the quality of micro-Doppler signatures at low SNRs.

\section{CONCLUSION}

The paper presents an effective method to augment measured spectrograms with more realistic synthetic spectrograms. We proposed a noise modeling framework based on GANs where the adversarial training learned the noise distribution directly from the measured spectrograms. We compared the classification performance of different data augmentation schemes under the following scenarios- simulation data with no noise, simulation data with added AWGN, and simulation data with MNM noise. The results showed that the data with MNM noise outperformed all other cases by $3 \%$ on an average. The performance is more pronounced, especially at low SNR scenarios (MNM $8.5 \%$ better than AWGN).

Overall, the paper demonstrates the feasibility of using more realistic simulated micro-Doppler spectrograms for data augmentation tasks. Future research will investigate the possibility of extracting multipath effects, occlusions directly from real spectrograms. We expect incorporating these factors into the simulated spectrograms could further improve the augmentation performance and enhance the overall training support.

\section{REFERENCES}

[1] World aging population. [Online]. Available: https: //www.un.org/en/development/desa/population/publications/pdf/ ageing/WPA2017_Highlights.pdf 
TABLE II: Classification accuracy results for augmentation study. $\mathrm{m}$ is kept fixed to $50 \%$. $\mathrm{s}$ is varied from $0 \%$ to $100 \%$ and SNR is varied from $0 \mathrm{~dB}$ to $21 \mathrm{~dB}$ with an interval of $3 \mathrm{~dB}$.

\begin{tabular}{|c|c|c|c|c|c|c|c|c|c|}
\hline \multicolumn{2}{|c|}{ Augmentation Study (Measurement data split 50\%-50\%) } & \multicolumn{8}{|c|}{ SNR(dB) } \\
\hline Percentage of Simulation Data Augmented & Noise Model & 0 & 3 & 6 & 9 & 12 & 15 & 18 & 21 \\
\hline \multirow{3}{*}{$100 \% \mathrm{M}+10 \% \mathrm{~S}$} & No Noise & 93.3 & 93.3 & 93.3 & 93.3 & 93.3 & 93.3 & 93.3 & 93.3 \\
\hline & AWGN & 91.6 & 89 & 92.7 & 93.1 & 93.4 & 93.1 & 94.2 & 93.1 \\
\hline & GAN Noise & 93.4 & 94.8 & 93.2 & 93.8 & 92.3 & 94.5 & 94.9 & 94.9 \\
\hline \multirow{3}{*}{$100 \% M+20 \% \mathrm{~S}$} & No Noise & 90.3 & 90.3 & 90.3 & 90.3 & 90.3 & 90.3 & 90.3 & 90.3 \\
\hline & AWGN & 90.9 & 89.7 & 90.7 & 91.9 & 93.4 & 92.4 & 93.6 & 93.4 \\
\hline & GAN Noise & 92.7 & 93.5 & 91.9 & 93.3 & 95.1 & 95 & 94.4 & 95.2 \\
\hline \multirow{3}{*}{$100 \% \mathrm{M}+30 \% \mathrm{~S}$} & No Noise & 91.8 & 91.8 & 91.8 & 91.8 & 91.8 & 91.8 & 91.8 & 91.8 \\
\hline & AWGN & 90.4 & 88.6 & 91.7 & 91.2 & 93.1 & 91.9 & 94.2 & 93.1 \\
\hline & GAN Noise & 89.4 & 92 & 93.6 & 91.2 & 94.6 & 94.4 & 93.3 & 94.6 \\
\hline \multirow{3}{*}{$100 \% \mathrm{M}+40 \% \mathrm{~S}$} & No Noise & 88.2 & 88.2 & 88.2 & 88.2 & 88.2 & 88.2 & 88.2 & 88.2 \\
\hline & AWGN & 89 & 89.8 & 90.9 & 90.9 & 90.8 & 94.2 & 91.6 & 93.6 \\
\hline & GAN Noise & 90.3 & 92.6 & 90.3 & 93.9 & 93.8 & 94.6 & 94.2 & 95.4 \\
\hline \multirow{3}{*}{$100 \% \mathrm{M}+50 \% \mathrm{~S}$} & No Noise & 90.7 & 90.7 & 90.7 & 90.7 & 90.7 & 90.7 & 90.7 & 90.7 \\
\hline & AWGN & 88.7 & 88.8 & 89.5 & 89.6 & 90.8 & 91.4 & 92.7 & 91.4 \\
\hline & GAN Noise & 90.2 & 87.9 & 93.3 & 92.3 & 93.5 & 93.2 & 94.7 & 94.8 \\
\hline \multirow{3}{*}{$100 \% M+60 \% \mathrm{~S}$} & No Noise & 89.0 & 89.0 & 89.0 & 89.0 & 89.0 & 89.0 & 89.0 & 89.0 \\
\hline & AWGN & 88.4 & 87.8 & 90.1 & 92.2 & 92.3 & 93 & 90.3 & 92.6 \\
\hline & GAN Noise & 90.3 & 89.3 & 93 & 93.3 & 93.2 & 93.7 & 93.6 & 94.5 \\
\hline \multirow{3}{*}{$100 \% \mathrm{M}+70 \% \mathrm{~S}$} & No Noise & 90.4 & 90.4 & 90.4 & 90.4 & 90.4 & 90.4 & 90.4 & 90.4 \\
\hline & AWGN & 88.3 & 86.5 & 90.7 & 90.1 & 91.5 & 90.7 & 92.9 & 92.3 \\
\hline & GAN Noise & 89.7 & 89.7 & 90.4 & 92.1 & 92.8 & 92.9 & 92.4 & 94.8 \\
\hline \multirow{3}{*}{$100 \% M+80 \% S$} & No Noise & 89.4 & 89.4 & 89.4 & 89.4 & 89.4 & 89.4 & 89.4 & 89.4 \\
\hline & AWGN & 88.1 & 86.7 & 90.2 & 89.8 & 90.4 & 92.6 & 90.2 & 93.5 \\
\hline & GAN Noise & 90.2 & 92.2 & 92.1 & 92.3 & 93.1 & 92.3 & 93.9 & 94.2 \\
\hline \multirow{3}{*}{$100 \% \mathrm{M}+90 \% \mathrm{~S}$} & No Noise & 91.1 & 91.1 & 91.1 & 91.1 & 91.1 & 91.1 & 91.1 & 91.1 \\
\hline & AWGN & 86.9 & 89.7 & 88.8 & 89.4 & 91.3 & 92 & 93.3 & 93.7 \\
\hline & GAN Noise & 85.2 & 90.7 & 92.1 & 92.4 & 92.2 & 91.1 & 93.4 & 95.4 \\
\hline \multirow{3}{*}{$100 \% \mathrm{M}+100 \% \mathrm{~S}$} & No Noise & 90.0 & 90.0 & 90.0 & 90.0 & 90.0 & 90.0 & 90.0 & 90.0 \\
\hline & AWGN & 86.7 & 89.3 & 87.1 & 90.1 & 91.1 & 89.9 & 90.8 & 91.6 \\
\hline & GAN Noise & 89.7 & 91.7 & 91.3 & 93.1 & 92.8 & 94.3 & 93.8 & 93.7 \\
\hline
\end{tabular}

[2] V. C. Chen, F. Li, S.-S. Ho, and H. Wechsler, "Analysis of micro-doppler signatures," IEE Proceedings-Radar, Sonar and Navigation, vol. 150, no. 4, pp. 271-276, 2003.

[3] B. Erol and M. G. Amin, "Radar data cube analysis for fall detection," in 2018 IEEE International Conference on Acoustics, Speech and Signal Processing (ICASSP). IEEE, 2018, pp. 2446-2450.

[4] F. Fioranelli, H. Li, J. Le Kernec, V. Busin, N. Jonsson, G. King, M. Tomlinson, and L. Viora, "Radar-based evaluation of lameness detection in ruminants: preliminary results," in 2019 IEEE MTT-S International Microwave Biomedical Conference (IMBioC), vol. 1. IEEE, 2019, pp. $1-4$.

[5] J. Le Kernec, F. Fioranelli, C. Ding, H. Zhao, L. Sun, H. Hong, J. Lorandel, and O. Romain, "Radar signal processing for sensing in assisted living: The challenges associated with real-time implementation of emerging algorithms," IEEE Signal Processing Magazine, vol. 36, no. 4, pp. 29-41, 2019.

[6] S. A. Shah and F. Fioranelli, "Rf sensing technologies for assisted daily living in healthcare: A comprehensive review," IEEE Aerospace and Electronic Systems Magazine, vol. 34, no. 11, pp. 26-44, 2019.

[7] E. Al Hadhrami, M. Al Mufti, B. Taha, and N. Werghi, "Transfer learning with convolutional neural networks for moving target classification with micro-doppler radar spectrograms," in 2018 International Conference on Artificial Intelligence and Big Data (ICAIBD). IEEE, 2018, pp. 148154.

[8] S. Zubeyde Gurbuz and M. G. Amin, "Radar-based human-motion recognition with deep learning: Promising applications for indoor monitoring," in Signal Processing Magazine. IEEE, 2019.

[9] S. Vishwakarma, W. Li, C. Tang, K. Woodbridge, R. Adve, and K. Chetty, "Simhumalator: An open source wifi based passive radar human simulator for activity recognition."

[10] C. Tang, S. Vishwakarma, W. Li, R. Adve, S. Julier, and K. Chetty, "Augmenting experimental data with simulations to improve activity classification in healthcare monitoring," in accepted for IEEE Radar Conference (RadarConf21). IEEE, 2021.

[11] Ni usrp 2921. [Online]. Available: http://sine.ni.com/nips/cds/view/p/ lang/en/nid/212995

[12] A. D. Singh, S. S. Ram, and S. Vishwakarma, "Simulation of the radar cross-section of dynamic human motions using virtual reality data and ray tracing," in 2018 IEEE Radar Conference (RadarConf18). IEEE, 2018, pp. 1555-1560.

[13] B. Erol and S. Z. Gurbuz, "A kinect-based human micro-doppler simulator," IEEE Aerospace and Electronic Systems Magazine, vol. 30, no. 5, pp. 6-17, 2015.

[14] Y. Lin, J. Le Kernec, S. Yang, F. Fioranelli, O. Romain, and Z. Zhao, "Human activity classification with radar: Optimization and noise robustness with iterative convolutional neural networks followed with random forests," IEEE Sensors Journal, vol. 18, no. 23, pp. 9669-9681, 2018.

[15] G. Li, R. Zhang, M. Ritchie, and H. Griffiths, "Sparsity-driven microdoppler feature extraction for dynamic hand gesture recognition," IEEE Transactions on Aerospace and Electronic Systems, vol. 54, no. 2, pp. 655-665, 2017.

[16] C. Tang, W. Li, S. Vishwakarma, K. Woodbridge, S. Julier, and K. Chetty, "Learning from natural noise to denoise micro-doppler spectrogram," 2021.

[17] I. Gulrajani, F. Ahmed, M. Arjovsky, V. Dumoulin, and A. C. Courville, "Improved training of wasserstein gans," Advances in neural information processing systems, vol. 30, pp. 5767-5777, 2017. 